\title{
Front Matter: Volume 9266
}

, "Front Matter: Volume 9266," Proc. SPIE 9266, High-Power Lasers and Applications VII, 926601 (19 December 2014); doi: 10.1117/12.2181445

SPIE. Event: SPIE/COS Photonics Asia, 2014, Beijing, China 


\title{
High-Power Lasers and Applications VII
}

\author{
Ruxin Li \\ Upendra N. Singh \\ Robert F. Walter \\ Editors
}

9-11 October 2014

Beijing, China

Sponsored by

SPIE

COS_Chinese Optical Society

Cooperating Organizations

Tsinghua University (China) • Peking University (China) • University of Science and Technology of China (China) • Zhejiang University (China) - Tianjin University (China) • Beijing Institute of Technology (China) • Beijing University of Posts and Telecommunications (China) - Nankai University (China) - Changchun University of Science and Technology (China) • University of Shanghai for Science and Technology (China) • Capital Normal University (China) Huazhong University of Science and Technology (China) • Beijing Jiaotong University (China) • Shanghai Institute of Optics and Fine Mechanics (China) - Changchun Institute of Optics and Fine Mechanics (China) - Institute of Semiconductors (China) • Institute of Optics and Electronics (China) • Institute of Physics (China) • Shanghai Institute of Technical Physics (China) - China Instrument and Control Society (China) - Optoelectronics Technology Committee, COS (China) • SPIE National Committee in China (China) • Optical Society of Japan (Japan) • Optical Society of Korea (Korea, Republic of) - The Australian Optical Society (Australia) • Optics and Photonics Society of Singapore (Singapore) • European Optical Society

Supporting Organizations

CAST_China Association for Science and Technology (China)

NSFC-National Nature Science Foundation (China)

Published by

SPIE

Volume 9266

Proceedings of SPIE 0277-786X, V. 9266

SPIE is an international society advancing an interdisciplinary approach to the science and application of light.

High-Power Lasers and Applications VII, edited by Ruxin Li, Upendra N. Singh,

Robert F. Walter, Proc. of SPIE Vol. 9266, 926601 - (c) 2014 SPIE

CCC code: $0277-786 X / 14 / \$ 18 \cdot$ doi: $10.1117 / 12.2181445$ 
The papers included in this volume were part of the technical conference cited on the cover and title page. Papers were selected and subject to review by the editors and conference program committee. Some conference presentations may not be available for publication. The papers published in these proceedings reflect the work and thoughts of the authors and are published herein as submitted. The publisher is not responsible for the validity of the information or for any outcomes resulting from reliance thereon.

Please use the following format to cite material from this book:

Author(s), "Title of Paper," in High-Power Lasers and Applications VII, edited by Ruxin Li, Upendra N. Singh, Robert F. Walter, Proceedings of SPIE Vol. 9266 (SPIE, Bellingham, WA, 2014) Article CID Number.

ISSN: 0277-786X

ISBN: 9781628413397

Published by

SPIE

P.O. Box 10, Bellingham, Washington 98227-0010 USA

Telephone +1 3606763290 (Pacific Time) · Fax +1 3606471445

SPIE.org

Copyright (@ 2014, Society of Photo-Optical Instrumentation Engineers.

Copying of material in this book for internal or personal use, or for the internal or personal use of specific clients, beyond the fair use provisions granted by the U.S. Copyright Law is authorized by SPIE subject to payment of copying fees. The Transactional Reporting Service base fee for this volume is $\$ 18.00$ per article (or portion thereof), which should be paid directly to the Copyright Clearance Center (CCC), 222 Rosewood Drive, Danvers, MA 01923. Payment may also be made electronically through CCC Online at copyright.com. Other copying for republication, resale, advertising or promotion, or any form of systematic or multiple reproduction of any material in this book is prohibited except with permission in writing from the publisher. The CCC fee code is 0277-786X/14/\$18.00.

Printed in the United States of America.

Publication of record for individual papers is online in the SPIE Digital Library.

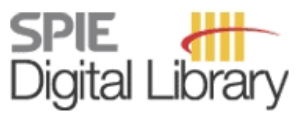

SPIEDigitallibrary.org

Paper Numbering: Proceedings of SPIE follow an e-First publication model, with papers published first online and then in print and on CD-ROM. Papers are published as they are submitted and meet publication criteria. A unique, consistent, permanent citation identifier (CID) number is assigned to each article at the time of the first publication. Utilization of CIDs allows articles to be fully citable as soon as they are published online, and connects the same identifier to all online, print, and electronic versions of the publication. SPIE uses a six-digit CID article numbering system in which:

- The first four digits correspond to the SPIE volume number.

- The last two digits indicate publication order within the volume using a Base 36 numbering

system employing both numerals and letters. These two-number sets start with 00, 01, 02, 03, 04, $05,06,07,08,09,0 A, 0 B \ldots$. 0Z, followed by 10-1Z, 20-2Z, etc.

The CID Number appears on each page of the manuscript. The complete citation is used on the first page, and an abbreviated version on subsequent pages. Numbers in the index correspond to the last two digits of the six-digit CID Number. 


\title{
Contents
}

\author{
vii Authors \\ ix Symposium Committees \\ xi Conference Committee
}

\section{SESSION $1 \quad$ ULTRAFAST LASERS}

926604 Temporal pulse cleaning by a self-diffraction process for ultrashort laser pulses [9266-3]

926605 An experimental method for pulse-width measurement in partial positions of an ultrashortpulsed beam [9266-4]

926606 Analysis of temporal contrast degradation due to wave front deviation in large aperture ultra-short pulse focusing system [9266-5]

\section{SESSION 2 HIGH POWER LASERS}

926607 Research and construction progress of SG-III laser facility (Invited Paper) [9266-6]

926608 Analysis on laser-induced thermoelastic deformation of DMs [9266-7]

926609 Ray-tracing method to analyze and quantify the light enhancement around subsurface defects in transparent materials [9266-8]

$92660 \mathrm{~A}$ Analysis of nonlinear self-focusing phenomenon in high-power laser system based on raytracing [9266-9]

9266 OB Design and comparison of laser windows for high-power lasers [9266-10]

$92660 \mathrm{C}$ Precise measurement of transmittance and reflectance for large aperture optics [9266-11]

SESSION $3 \quad$ FIBER LASERS

9266 OD Optical properties of high power S-band fiber oscillators and amplifiers [9266-12]

$9266 \mathrm{OE} \quad$ Fiber laser at $\mathbf{2} \boldsymbol{\mu m}$ for soft tissue surgery [9266-13]

9266 OF 780W narrow linewidth all fiber laser with sinusoidal phase modulation [9266-14]

9266 OG Powerful 2- 
$9266 \mathrm{OH}$ A compact pulse-tunable fiber laser based on pulse-pumped high gain cavity [9266-16]

$926601 \quad$ Towards high-power random fiber laser [9266-17]

\section{SESSION 4 HIGH FIELD LASER PHYSICS}

9266 OM Research on chirped pulse stimulated Raman scattering in ethanol [9266-21]

\section{SESSION 5 DIODE PUMPED LASERS}

$92660 \mathrm{~N}$ Research of quasi-three-level thermal effect of diode-pumped Tm:YAG crystal [9266-22]

$926600 \quad$ High power 1060-nm super large vertical cavity semiconductor lasers [9266-23]

9266 OP Investigation of physical features of both static and flowing-gas diode-pumped rubidium vapor lasers [9266-24]

9266 OR $101 \mathrm{~W}$ quasi-continuous wave $\mathrm{Nd:YVO} 4$ slab laser with high beam quality [9266-26]

9266 OS Theoretical analysis of wavelength switchable diode-side-pumped Tm:YAG laser [9266-27]

\section{SESSION 6 LASER APPLICATIONS}

9266 OT Femtosecond laser microchannels fabrication based on electrons dynamics control using temporally or spatially shaped pulses (Invited Paper) [9266-28]

9266 OV Mid-ultraviolet pulsed laser micromachining of SiC [9266-30]

\section{SESSION 7 OTHER LASERS AND APPLICATIONS I}

$92660 X \quad$ Recent progress of the Integration Test Bed [9266-41]

9266 OY Injecting parameters design and performance test of the pre-igniter for continuous wave DF/HF chemical lasers [9266-33]

$92660 Z$ Characteristic optimization of 1.55- $\mu \mathrm{m}$ InGaAsP/InP high-power diode laser [9266-34]

926610 Broadband generation by multiple four-wave mixing process due to ASE Q-switching in high-power double-clad ytterbium-doped fiber amplifier [9266-35]

\section{SESSION 8 OTHER LASERS AND APPLICATIONS II}

926613 Investigation on the formation of second-order-hot-image-like fringes [9266-38]

926614 Finite element simulation of laser-induced guided wave in layered structure [9266-39] 
926615 Numerical simulation of laser induced interfacial wave on the transparent solid/solid interface [9266-40]

926616 Spectral broadening and inhibition of amplitude and frequency modulation in Nd: glass regenerative amplifier [9266-42]

926617 Wave front aberration analysis of slit spatial filtering system [9266-43]

926618 Volume-discharge formed in $\mathrm{SF}_{6}$ and $\mathrm{C}_{2} \mathrm{H}_{6}$ mixtures without preionization [9266-44]

926619 Experimental comparison of the cutting speed and quality for mild and stainless steel sheets with fiber and $\mathrm{CO}_{2}$ lasers [9266-45]

9266 lA Influence trend of temperature distribution in skin tissue generated by different exposure dose pulse laser [9266-46]

9266 1B Efficient bending compensation of large mode area fiber [9266-47]

9266 1E High-power continuous-wave ytterbium-doped fiber oscillator at $1018 \mathrm{~nm}$ [9266-50]

9266 IF All-fiber designed narrow line-width $1.55 \mu \mathrm{m}$ double cladding fiber lasers [9266-51]

9266 IG High-power high-efficiency picosecond $355 \mathrm{~nm}$ ultraviolet laser based on $\mathrm{La}_{2} \mathrm{CaB}_{10} \mathrm{O}_{19}$ crystal [9266-52]

926611 Pump couplers in a series connection [9266-54]

$92661 \mathrm{~J}$ Uniformity of pump intensity distribution in diode-array side-pumped laser rod [9266-55]

9266 1K Design and fabricate large scale gold coated pulse compression grating for Ti: sapphire laser [9266-56]

$9266 \mathrm{lL}$ Analysis of the refractive index change of optical waveguide in $\mathrm{LiNbO}_{3}$ using a femtosecond laser [9266-57]

9266 1M Fabrication of lithium niobate-based low-loss bend optical waveguide [9266-58]

9266 IN Design of high-efficiency broad-bandwidth pulse compression device based on composite transmission grating with high damage threshold [9266-59]

926610 Characteristics of nonlinear imaging of broadband laser stacked by chirped pulses [9266-60]

9266 IP Development of a sub-petawatt ultrashort laser facility [9266-61]

9266 IR Analysis of chirped-pulse-amplification system based on Offner triplet stretcher and transmission grating compressor [9266-63] 
$92661 \mathrm{U}$ Longitudinally excited $\mathrm{CO}_{2}$ laser with tail-free short pulse [9266-66]

9266 IV Simulation of wavefront sensorless correction based on Stochastic Parallel Gradient Descent algorithm [9266-67] 


\title{
Authors
}

Numbers in the index correspond to the last two digits of the six-digit citation identifier (CID) article numbering system used in Proceedings of SPIE. The first four digits reflect the volume number. Base 36 numbering is employed for the last two digits and indicates the order of articles within the volume. Numbers start with 00, 01, 02, 03, 04, 05, 06, 07, 08, 09, OA, OB...0Z, followed by 10-1Z, 20-2Z, etc.

\author{
Akitsu, Tetsuya, $1 \mathrm{U}$ \\ An, Guofei, OP \\ $\mathrm{Bi}$, Yong, $1 \mathrm{E}$ \\ Cai, He, OP \\ Cen, Zhaofeng, OA \\ Chang, Zenghu, 1K, IN \\ Chen, Dehuai, OX \\ Chen, Dongxian, $\mathrm{OC}$ \\ Chen, Ji, OX \\ Chen, Jun-jiang, 1L, 1M \\ Chen, Liezun, 10 \\ Chen, Lin, OX \\ Chen, LU, OR \\ Chen, Nianjiang, OF \\ Chen, Xiao, 11 \\ Chen, Xinrong, 1K, 1N, IR \\ Chen, Yuanbin, 07 \\ Chowdhury, Sourav D., 10 \\ Dai, Zhiping, 10 \\ Dang, Zhao, 07 \\ Deng, Xuewei, 07 \\ Ding, Lei, 07 \\ Dobashi, Kazuma, $1 \mathrm{U}$ \\ Fan, Ya-Xian, 1B \\ Fang, Xiaoting, OY \\ Farida, Souaci, 1B \\ Feng, Bin, 07 \\ Feng, Jie, 1L, 1M \\ Feng, Xiaobo, $1 \mathrm{E}$ \\ Fu, Xiquan, 05 \\ Fu, Xuejun, OX \\ Gao, Dong, OX \\ Gao, Qi, 06 \\ Ghosh, Aditi, OE \\ Golishev, A. A., 19 \\ Gong, Mali, OD, 11 \\ Gong, Wupeng, $1 \mathrm{E}$ \\ Guo, Ailin, 06 \\ Guo, Liangfu, 07 \\ Guo, Xiao-Yang, OM \\ Guo, Yi, 04, IP \\ Han, Juhong, OP \\ Han, Wei, OX \\ Hao, Jinping, OD, OF \\ He, Guotan, 17 \\ Hu, Dongxia, 07 \\ $\mathrm{Hu}$, Jie, OT \\ Hu, Jinping, OV \\ Hu, Yonghua, 13
}

\author{
Hua, Neng, 09 \\ Huang, Bing, $O Y$ \\ Huang, Xiaojun, IP \\ Hui, Hongchao, OC \\ Ji, Chen, 0O, OZ \\ Jiang, Dongbin, $1 \mathrm{P}$ \\ Jiang, Lan, OT \\ Jiang, Zongfu, 08 \\ Jin, Guangyong, 11 \\ Jin, Xiaoxi, OG \\ Jing, Feng, 07, OX \\ Jitsuno, Takahisa, $1 \mathrm{U}$ \\ Kang, Jun, 06 \\ $\mathrm{Ke}$, Changjun, 18 \\ Ke, Qing, $O Z$ \\ Leng, Yu-Xin, OM \\ Li, Chaoming, 1K, 1N, IR \\ Li, Guohui, 13 \\ Li, Jiaqiang, $1 \mathrm{G}$ \\ Li, Kai, IG \\ Li, Lin, 1N, 1R \\ $\mathrm{Li}$, Mingxing, OV \\ Li, Qing, 04, IP \\ Li, Ru-Xin, OM \\ Li, Sen, OX \\ Li, Xiaotong, OA \\ Li, Xiaowei, OT \\ Li, Xin, 1M \\ Li, Xuechun, 16 \\ Li, Yan-Yan, OM \\ Li, Yao, OD, OF \\ Li, Zhongxiao, $1 \mathrm{G}$ \\ Liang, Xingbo, OR \\ Liang, Yue, $O X$ \\ Lin, Donghui, 07 \\ Lin, Haipeng, OV \\ Lin, Honghuan, OX \\ Lin, Xuechun, $1 G$ \\ Lin, Xue-song, 1L, 1M \\ Lin, Zunqi, 09 \\ Ling, Xiaohui, 10 \\ Liu, Ang, 1M \\ Liu, Chunsheng, OV \\ Liu, De'an, OC \\ Liu, Haixia, OB, $1 \mathrm{~J}$ \\ Liv, Lanqin, 07 \\ Liu, Lei, OR \\ Liu, Linyue, 1N, $1 R$ \\ Liu, Pengjun, OT
}


Liu, Pengxiang, $1 \mathrm{G}$

Liu, Shanchao, OR

Liu, Wenguang, 08

Liu, Wenwen, OB, ON, OS, $1 \mathrm{~J}$

Liv, Xia, 1 A

Liu, Yang, OR

Liv, Yan-Qi, OM

Liu, Zejin, OG

Lu, Dan, 0O, $0 Z$

Lu, Shizhuan, 10

Lu, Xiao-Ming, OM

Lu, Yongfeng, OT

LU, Zhiming, $\mathrm{OH}$

LV, Haibin, Ol

Malikov, A. G., 19

Man, Da, OB, ON, OS, IJ

Miao, Jie, $O C$

Niu, Haisha, OB, ON, $1 \mathrm{~J}$

Niu, Yanxiong, OB, ON, OS, 1J

Orishich, A. M., 19

Pal, Atasi, OE

Pal, Debasis, OE

Pal, Mrinmay, 10

Pan, Xue, 16

Peng, Zhitao, 07

Qi, Litao, OV

Qiu, Yu, 1M

Saha, Maitreyee, 10

Sen, Ranjan, OE, 10

Shan, Faxian, $1 G$

Shan, Ning, 1 A

Shao, Ping, 09

Shekhar, Nishant, 10

Shen, Yi, 13

Shulyatyev, V. B., 19

Su, Hongxin, IF

Su, Jingqin, 04, $1 P$

Sun, Li, 04, 1P

Tan, Chao, 05

Tan, Shaoyang, 00, $0 Z$

Tang, Jun, OX

Tang, Qingju, OV

Tang, Xiaojun, OR

Uno, Kazuyuki, $1 \mathrm{U}$

Wan, Hongdan, $\mathrm{OH}$

Wang, Baohua, $1 \mathrm{G}$

Wang, Caili, OB, ON, OS, $1 \mathrm{~J}$

Wang, Cheng, OM

Wang, Gang, IV

Wang, Hongyuan, OP

Wang, Jiangfeng, 16

Wang, Jianjun, 07

Wang, Jin, $\mathrm{OH}$

Wang, Jingli, $\mathrm{OH}$

Wang, Lirong, $1 G$

Wang, Nan, $1 G$

Wang, Wei, 00

Wang, Weiwei, OA

Wang, Wenyi, OX

Wang, Xiaodong, 04, IP
Wang, Xiaolin, OG, Ol

Wang, Xiong, OG

Wang, Xiongfei, OF

Wang, YOu, OP

Wang, Youwen, 10

Wang, Yuye, $1 \mathrm{G}$

Wang, Zhe, OR

Wang, Zhijing, $1 \mathrm{~A}$

Wei, Binbin, 08

Wei, Xiaofeng, 07, OX

Wu, Jianhong, 1K, 1N, 1R

Wu, Rong, 09

Wu, Yicheng, $1 G$

Wu, Zhiyang, $1 \mathrm{~F}$

Xia, Bo, OT

Xia, Yanwen, OX

Xiao, Hu, OG, OI

Xiao, Qirong, 11

Xie, Kun, 08

Xie, Na, 04, $1 \mathrm{P}$

Xie, Xinglong, 06

Xie, Yujiang, $0 \mathrm{C}$

Xiong, Han, 17

$X U$, Degang, $1 G$

$X \cup$, Lijing, $1 F$

$X u$, Xiaojun, Ol

$X u, Y i, O M$

$X \cup e$, Liangping, $\mathrm{OP}$

Xue, Liping, 14, 15

Xue, Yuhao, $1 \mathrm{E}$

Yan, Chao, $1 G$

Yan, Feiyue, $\mathrm{OX}$

Yan, Ping, OD, 11

Yan, Xueliang, OT

Yang, Lijia, OY

Yang, Lin, $O C$

Yang, Pengqian, $0 \mathrm{C}$

Yang, Qingwei, 06

Yang, Shi-han, 1L, 1M

Yang, Ying Ying, $1 G$

Yao, Jianquan, $1 \mathrm{G}$

You, Kaiming, 10

Yu, Haijuan, $1 G$

Yuan, Li-Bo, 1B

Yuan, Shengfu, OY

Yuan, Xiao, 17

Zeng, Xiaoming, IP

Zhai, Teng, $00,0 Z$

Zhang, Dayong, OD, OF

Zhang, Ge, 18

Zhang, Guochun, 1G

Zhang, Hanwei, 0G, Ol

Zhang, Hong, $1 \mathrm{E}$

Zhang, Kun, OF

Zhang, Lei, 09

Zhang, Liang, $1 \mathrm{~L}$

Zhang, Liming, OF

Zhang, Ling, IG

Zhang, Luwei, OA

Zhang, Renli, IE 
Zhang, Ruikang, $0 \mathrm{O}, 0 \mathrm{Z}$

Zhang, Shaobo, 13

Zhang, Shujuan, 18

Zhang, Wei, OP

Zhang, Xiang, 17

Zhang, Yuqi, 16

Zhao, Dongfeng, 09

Zhao, Hong, OD, OF, OR

Zhao, Junpu, OX

Zhao, Yan, 14, 15

Zheng, Kuixing, OX

Zheng, Wanguo, 07, 0X

Zhou, Kainan, 04, 1P

Zhou, Pu, OG, Ol

Zhou, Qiong, 08

Zhou, Shouhuan, OF, OR

Zhou, Wei, 07

Zhou, $\mathrm{Yu}, \mathrm{OH}$

Zhou, Zi-gang, 1L, 1M

Zhu, Baoqiang, OC

Zhu, Chen, OD, OF

Zhu, Haidong, 06

Zhu, Jianqiang, 06, OC

Zhu, Ping, 06

Zhu, Qihua, 07, 0X, 1P

Zhuang, Zhenwu, 17

Zou, Kuaisheng, 17

Zou, Xiao, OM

Proc. of SPIE Vol. $9266926601-9$

Downloaded From: https://www.spiedigitallibrary.org/conference-proceedings-of-spie on 26 Apr 2023 Terms of Use: https://www.spiedigitallibrary.org/terms-of-use 
Proc. of SPIE Vol. $9266926601-10$

Downloaded From: https://www.spiedigitallibrary.org/conference-proceedings-of-spie on 26 Apr 2023 Terms of Use: https://www.spiedigitallibrary.org/terms-of-use 


\title{
Symposium Committees
}

\author{
General Chairs
}

H. Philip Stahl, NASA Marshall Space Flight Center (USA)

Bingkun Zhou, Tsinghua University (China)

General Co-chairs

Arthur Chiou, National Yang-Ming University (Taiwan, China)

Jianlin Cao, China Ministry of Science and Technology (China)

Junhao Chu, Shanghai Institute of Technical Physics (China)

Technical Program Chairs

Songlin Zhuang, Shanghai University of Science and Technology (China)

Xingde Li, Johns Hopkins University (United States)

Technical Program Co-chairs

Qiming Wang, Institute of Semiconductors (China)

Xu Liu, Zhejiang University (China)

Daoyin Yu, Tianjin University (China)

Qihuang Gong, Peking University (China)

Tianchu Li, National Institute of Metrology (China)

Wei Huang, Nanjing University of Posts and Telecommunications (China)

Local Organizing Committee Chair

Guangcan Guo, University of Science and Technology of China (China) 
Local Organizing Committee Co-chairs

Guoqiang Ni, Beijing Institute of Technology (China)

Shusen Xie, Fujian Normal University (China)

Xiaomin Ren, Beijing University of Posts and Telecommunications (China)

Ying Gu, People's Liberation Army General Hospital (China)

Huilin Jiang, Changchun University of Science and Technology (China)

General Secretary

Qihuang Gong, Peking University (China)

Local Organizing Committee

Yan Li, Chinese Optical Society/Peking University (China)

Zhiping Zhou, Peking University (China)

Changhe Zhou, Shanghai Institute of Optics and Fine Mechanics (China)

Qingming Luo, Huazhong University of Science and Technology (China)

Chongxiu Yu, Beijing University of Posts and Telecommunications (China)

Hongda Chen, Institute of Semiconductors (China)

Yongtian Wang, Beijing Institute of Technology (China)

Yiping Cui, Southeast University (China)

Xuping Zhang, Nanjing University (China)

Feijun Song, Daheng Corporation (China)

Cunlin Zhang, Capital Normal University (China)

Yanting Lu, Nanjing University (China)

Yuejin Zhao, Beijing Institute of Technology (China)

Chunqing Gao, Beijing Institute of Technology (China)

Tiegen Liu, Tianjin University (China)

Xiaocong Yuan, Nankai University (China)

Weimin Chen, Chongqing University (China)

Zhongwei Fan, Academy of Optoelectronics (China)

Hanyi Zhang, Tsinghua University (China)

Lan Wu, Zhejiang University (China)

Yongsheng Zhang, University of Science and Technology of China (China)

Hong Yang, Peking University (China)

Xiaoying Li, Tianjin University (China)

Wei Xiong, Chinese Optical Society (China) 


\title{
Conference Committee
}

\author{
Conference Chairs
}

Ruxin Li, Shanghai Institute of Optics and Fine Mechanics (China) Upendra N. Singh, NASA Langley Research Center (United States) Robert F. Walter, Schafer Corporation (United States)

\section{Conference Program Committee}

Willy L. Bohn, BohnLaser Consult (Germany)

Robert L. Byer, Stanford University (United States)

Dianyuan Fan, Hunan University (China)

Tomoo Fujioka, Tokai University (Japan)

Mali Gong, Tsinghua University (China)

Shibin Jiang, AdValue Photonics, Inc. (United States)

Do-Kyeong Ko, Gwangju Institute of Science and Technology

(Korea, Republic of)

Zejin Liu, National University of Defense Technology (China)

DeYuan Shen, Fudan University (China)

Yi Su, Institute of Applied Electronics (China)

Shuangchun Wen, Hunan University (China)

Zuyan Xu, Technical Institute of Physics and Chemistry (China)

Jianquan Yao, Tianjin University (China)

Tai Hyun Yoon, Korea University (Korea, Republic of)

Jirong Yu, NASA Langley Research Center (United States)

Heping Zeng, East China Normal University (China)

Xiaomin Zhang, China Academy of Engineering Physics (China)

Shouhuan Zhou, North China Research Institute of Electro-optics (China)

\section{Session Chairs}

1 Ultrafast Lasers

Zhiyi Wei, Institute of Physics (China)

2 High Power Lasers

Yulei Wang, Harbin Institute of Technology (China)

3 Fiber Lasers

Yulei Wang, Harbin Institute of Technology (China)

4 High Field Laser Physics

Jie Hu, Beijing Institute of Technology (China) 
5 Diode Pumped Lasers

Hong Zhao, North China Research Institute of Electro-optics (China)

6 Laser Applications

Xin Ding, Tianjin University (China)

7 Other Lasers and Applications I

Degang Xu, Tianjin University (China)

8 Other Lasers and Applications II

Degang Xu, Tianjin University (China) 